\title{
Математическая модель конкуренции политических партий
}

\author{
1) Ганичева А.В., ${ }^{2)}$ Ганичев А.B. \\ 1) Тверская государственная сельскохозяйственная академия, Россия, \\ 170904, Тверь, ул. Маршала Василевского, 7 \\ 2) Тверской государственный технический университет, Россия, \\ 170026, Тверь, наб. Аф. Никитина, 22 \\ E-mail: alexej.ganichev@yandex.ru
}

\begin{abstract}
Аннотация. Одной из важнейших проблем общественного развития является организация конкуренции (борьбы) политических партий. Для анализа и прогнозирования данного процесса наиболее удобен метод математического моделирования. Актуальность разработки модели борьбы партий определяется важностью рассматриваемого процесса для определения стратегии жизни стран и народов. В данном исследовании для описания конкуренции партий используется модифицированная модель Ричардсона (гонки вооружений) в виде динамической системы дифференциальных уравнений. С помощью операторного метода получено аналитическое решение динамической системы дифференциальных уравнений. Для пояснения разработанного метода рассмотрен конкретный числовой пример. Проанализированы особенности применения модели конкуренции политических партий. Определены перспективы дальнейшего развития нового разработанного метода.
\end{abstract}

Ключевые слова: мероприятия, система дифференциальных уравнений, коэффициенты агитируемости, коэффициенты выбытия, операторная система, оригинал, изображение.

Для цитирования: Ганичева А.В., Ганичев А.В. 2021. Математическая модель конкуренции политических партий. Экономика. Информатика, 48 (2): 341-349. DOI 10.52575/2687-0932-2021-48-2-341-349.

\section{Mathematical model of political party competition}

\author{
1) Antonina V. Ganicheva, ${ }^{2)}$ Alexey V. Ganichev \\ 1) Tverskaya state agricultural Academy, 7 Marshal Vasilevsky St, Tver, 170904, Russia \\ ${ }^{2)}$ Tverskoy state technical University, 22 nab. AF. Nikitin, Tver, 170026, Russia \\ E-mail: alexej.ganichev@yandex.ru
}

\begin{abstract}
One of the most important problems of social development is the organization of competition (struggle) of political parties. For the analysis and forecasting of this process, the most convenient method is mathematical modeling. The relevance of the development of a model of the struggle of parties is determined by the importance of the process under consideration for determining the strategy of the life of countries and peoples. In this study, a modified Richardson model (arms race) in the form of a dynamic system of differential equations is used to describe the competition of parties. An analytical solution of a dynamic system of differential equations is obtained using the operator method. To explain the developed method, a specific numerical example is considered. The features of the application of the model of competition of political parties are analyzed. The prospects for further development of the new developed method are determined. The developed method can be used for the analysis of interethnic, religious conflicts, for determining the maturation of an explosive, crisis situation in society. It allows you to analyze the clash of views and interests of individuals.
\end{abstract}

Keywords: events, system of differential equations, agitability coefficients, retirement coefficients, operator system, original, image.

For citation: Ganicheva A.V., Ganichev A.V. 2021. Mathematical model of political party competition. Economics. Information technologies, 48 (2): 341-349. (in Russian). DOI 10.52575/2687-0932-2021-48-2341-349. 


\section{Введение}

Для успешного общественного развития необходимо проводить исследования социальных процессов и явлений с целью их анализа и прогнозирования. При этом возникает проблема обработки и анализа больших массивов информации. Для выделения, формализации результативных признаков и существенных факторов наиболее удобным является метод математического моделирования. Актуальность применения этого метода для исследования социально-экономических процессов определяется цифровизацией -внедрением цифровых технологий в разные сферы жизни общества. При исследовании социальных процессов методом моделирования следует учитывать динамичность исследуемых процессов. Разнообразие социальных процессов вызывает необходимость применения большого количества адекватных им математических моделей, использующих современные математические методы.

Примеры использования оптимального управления в социальных моделях приведены в обзоре [Comissiong, Sooknanan, 2018]. В работе [Андрианова и др., 2020] для описания процессов в сложных системах с присутствием человеческого фактора используются нестационарные временные ряды. Многие социальные процессы связаны с конфликтными ситуациями и противоборством государств, народов, религиозных общин, партий, группировок и т. д. Противоборство может протекать в форме вооруженных конфликтов, экономической конкуренции, информационного противоборства, политической борьбы. Для описания моделей динамического противоборства в настоящее время наиболее часто применяют модели в виде систем дифференциальных уравнений [Rinaldi, Rossa, 2018, Tsybulin, Khosaeva, 2019]. Так, в статьях [Михайлов и др., 2017, Петров, Прончева, 2019, Петров, 2017, Marevtseva, 2017] для исследования вопросов информационного противоборства в социуме применяются системы нелинейных дифференциальных уравнений. Для исследования противоборства партий, групп успешно применяются динамические модели, описывающие развитие некоторого процесса во времени: Лотки - Вольтерры (хищник-жертва) [Dominioni et al., 2018], Ричардсона (гонка вооружений) [Канищева, 2017], Мисры (эпидемиологический подход) [Misra, Kumar, 2012, Nyabadza et al., 2016, Winkel, 201720], диффузии Ланжевена [Petukhov et al., 2018]. Для решения конфликтных ситуаций в социальной сфере применяются также модели теории игр [Остапенко и др., 2012, Kubiv et al., 2020]. Существует большое количество разнообразных моделей партий [Гильманов, 2017].

Для жизни современного общества большое значение имеет конкурентная борьба политических партий. Несмотря на то, что в настоящее время разработано большое количество математических моделей социальных процессов, проблема моделирования конкурентной борьбы политических партий является недостаточно изученной. Актуальность данной темы с развитием общества только возрастает. Необходимо разработать наглядную модель партийной конкуренции, позволяющую получить аналитическое решение задачи, чтобы не привлекать сложные и громоздкие численные методы, требующие больших затрат вычислительных ресурсов. Результаты, полученные с помощью численных методов, в ряде случаев трудно поддаются анализу.

Одной из наиболее распространенных моделей для описания вооруженных конфликтов является модель Ричардсона [Руренко, 2017, Фирсова, Глухова, 2015]. В данном исследовании для описания процесса конкуренции (борьбы) политических партий применяется модифицированная модель Ричардсона. В разработанной модели не используются параметры, характеризующие претензии сторон, т. к. они не характерны для борьбы партий.

В разделе «Материалы и Методы» данного исследования разработана модель борьбы партий. В разделе «Результаты и Дискуссия» на конкретном примере показана возможность применения разработанного метода и приведены его особенности. 


\section{Материалы и методы} партий.

Целью работы является разработка математической модели борьбы политических

Для реализации поставленной цели необходимо решить следующие задачи:

1) формализовать динамическую модель конкуренции политических партий в виде системы дифференциальных уравнений;

2) получить аналитическое решение системы;

3) для проверки разработанного алгоритма рассмотреть числовой пример;

4) проанализировать особенности применения и дальнейшего развития нового разработанного метода описания конкуренции политических партий.

Рассмотрим и формализуем постановку проблемы.

Пусть имеется две партии, и в момент времени t у первой партии $x=x(t)$ сторонников, у второй $-y=y(t)$ сторонников. Во время различного рода мероприятий (дебаты, дискуссии, праймериз, демонстрации, митинги, шествия, съезды) количество $x$ и $y$ меняется, и этот процесс можно описать системой линейных дифференциальных уравнений:

$$
\left\{\begin{array}{l}
x^{\prime}=\alpha y+\gamma x, \\
y^{\prime}=\beta x+\delta y,
\end{array}\right.
$$

$x\left(t_{0}\right)=x_{0}, y\left(t_{0}\right)=y_{0}$, здесь $t_{0}-$ начальное время.

Сначала рассмотрим случай, когда $x^{\prime}\left(t_{0}\right) \neq 0$ и $y^{\prime}\left(t_{0}\right) \neq 0$.

Здесь $x^{\prime}, y^{\prime}$ - соответственно скорости изменения числа $x$ и $y, \alpha, \beta, \gamma, \delta$ коэффициенты пропорциональности, причем $\alpha>0, \beta>0$ (коэффициенты агитируемости), $\gamma<0, \delta<0$ (коэффициенты выбытия). В самом деле, каждая партия изменяет скорость роста (или сокращения) агитационной работы, пропорционально уровню числа сторонников другой партии, т. е.

$$
\left\{\begin{array}{l}
x^{\prime}=\alpha y, \\
y^{\prime}=\beta x .
\end{array}\right.
$$

В то же время, чем больше число сторонников данной партии, тем меньше скорость его роста, т. е.

$$
\left\{\begin{array}{l}
x^{\prime}=\gamma x, \\
y^{\prime}=\delta y .
\end{array}\right.
$$

Получим оценку для коэффициентов $\alpha, \beta, \gamma, \delta$ на примере оценки $\gamma$. При условии отсутствия сторонников второй партии из первого уравнения системы (3) находим: $x(t)=e^{\gamma\left(t-t_{0}\right)} x\left(t_{0}\right)$. Отсюда $x\left(t_{0}-\frac{1}{\gamma}\right)=x\left(t_{0}\right) \frac{1}{e}$, т.е. $-\frac{1}{\gamma}-$ это время, которое требуется для снижения количества сторонников первой партии в $e=2,7$ раз. Аналогично $-\frac{1}{\delta}-$ время для снижения количества сторонников второй партии при отсутствии сторонников первой партии, $\frac{1}{\alpha}\left(\frac{1}{\beta}\right)$ - время для увеличения числа сторонников первой (второй) партии в $e=2,7$ раз при их отсутствии.

Решим систему (1) операторным методом.

Рассматривая функции $x(t)$ и $y(t)$ как оригиналы, введем их изображения:

$$
x(t)=X(p), y(t)==Y(p) .
$$


Записываем изображения производных:

$$
x^{\prime}(t)=p X(p)-x_{0}, y^{\prime}==p Y(p)-y_{0} .
$$

Операторная система:

$$
\left\{\begin{array}{l}
p X(p)-x_{0}=\alpha Y(p)+\gamma X(p), \\
p Y(p)-y_{0}=\beta X(p)+\delta Y(p),
\end{array}\right.
$$

T. e.

$$
\left\{\begin{array}{c}
(p-\delta) X(p)-\alpha Y(p)=x_{0} \\
-\beta X(p)+(p-\delta) Y(p)=y_{0}
\end{array}\right.
$$

Решаем эту систему:

$$
\begin{gathered}
\Delta=\left|\begin{array}{cc}
p-\gamma & -\alpha \\
-\beta & p-\delta
\end{array}\right|=(p-\gamma)(p-\delta)-\alpha \beta=p^{2}-p(\delta+\gamma)+\gamma \delta-\alpha \beta ; \\
\Delta X(p)=\left|\begin{array}{cc}
x_{0} & -\alpha \\
y_{0} & p-\delta
\end{array}\right|=x_{0}(p-\delta)-\alpha y_{0} ; \Delta X(p)=\left|\begin{array}{cc}
p-\gamma & x_{0} \\
-\beta & y_{0}
\end{array}\right|=y_{0}(p-\gamma)+\beta x_{0} .
\end{gathered}
$$

Отсюда получаем

$$
\left\{\begin{array}{l}
X(p)=\frac{x_{0}(p-\delta)+\alpha y_{0}}{p^{2}-p(\delta+\gamma)+\gamma \delta-\alpha \beta} \\
Y(p)=\frac{y_{0}(p-\gamma)+\beta x_{0}}{p^{2}-p(\delta+\gamma)+\gamma \delta-\alpha \beta} .
\end{array}\right.
$$

Преобразуем $X(p)$ и $Y(p)$. Получим:

$$
\left\{\begin{array}{l}
X(p)=\frac{x_{0}(p-\delta)}{p^{2}-p(\delta+\gamma)+\gamma \delta-\alpha \beta}+\frac{\alpha y_{0}}{p^{2}-p(\delta+\gamma)+\gamma \delta-\alpha \beta}, \\
Y(p)=\frac{y_{0}(p-\gamma)}{p^{2}-p(\delta+\gamma)+\gamma \delta-\alpha \beta}+\frac{\beta x_{0}}{p^{2}-p(\delta+\gamma)+\gamma \delta-\alpha \beta} .
\end{array}\right.
$$

Выделим в знаменателе полный квадрат:

$$
\begin{aligned}
A= & p^{2}-p(\delta+\gamma)+\gamma \delta-\alpha \beta=p^{2}-2 \cdot \frac{1}{2} p(\delta+\gamma)+\frac{1}{4}(\delta+\gamma)^{2}-\frac{1}{4}(\delta+\gamma)^{2}+\gamma \delta-\alpha \beta= \\
& =\left(p-\frac{1}{2}(\delta+\gamma)\right)^{2}-\frac{1}{4}(\delta+\gamma)^{2}+\gamma \delta-\alpha \beta=\left(p-\frac{1}{2}(\delta+\gamma)\right)^{2}-\frac{1}{4}(\delta-\gamma)^{2}-\alpha \beta .
\end{aligned}
$$

Пусть

$$
B^{2}=\frac{1}{4}(\delta+\gamma)^{2}-\gamma \delta+\alpha \beta
$$

Тогда $A=\left(p-\frac{1}{2}(\delta+\gamma)\right)^{2}-B^{2}$.

Для $B$ возможны следующие случаи:

1) $\frac{1}{4}(\delta-\gamma)^{2}+\alpha \beta \leq 0$. Такая ситуация возможна тогда и только тогда, когда $\gamma=\delta$ и либо $\alpha=0$, либо $\beta=0$, и левая часть равна 0 , при этом $A=(p-\delta)^{2}, B=0$. Пусть, например, $\alpha=0$, тогда первое слагаемое из (4) для $X(p)$ преобразуется так:

$$
\frac{x_{0}(p-\delta)}{A}==x_{0} e^{\delta t} \cdot \cos B t=x_{0} e^{\delta t},
$$

второе слагаемое 


$$
\frac{\alpha y_{0}}{(p-\delta)^{2}}=\alpha y_{0} t e^{\delta t}
$$

2) $\frac{1}{4}(\delta-\gamma)^{2}+\alpha \beta>0$.

Для случая 2) преобразуем числитель первого слагаемого в $X(p)$ системы (4):

$$
\begin{gathered}
x_{0}(p-\delta)=x_{0} p-x_{0} \delta-x_{0} \cdot \frac{1}{2}(\delta+\gamma)+x_{0} \cdot \frac{1}{2}(\delta+\gamma)=x_{0}\left(p-\frac{1}{2}(\delta+\gamma)\right)-x_{0}\left(\delta-\frac{1}{2}(\delta+\gamma)\right)= \\
=x_{0}\left(p-\frac{1}{2}(\delta+\gamma)-\frac{1}{2} x_{0}(\delta-\gamma) .\right.
\end{gathered}
$$

Тогда первое слагаемое преобразуется к виду:

$$
\frac{x_{0}\left(p-\frac{1}{2}(\delta+\gamma)\right)}{A}==x_{0} e^{\frac{1}{2}(\delta+\gamma) t} \cdot \mathrm{chBt} .
$$

Прибавив ко второму слагаемому в $X(p)$ системы (4) второе слагаемое из (8), деленное на $A$, получим: $C=\frac{\alpha y_{0}-\frac{1}{2} x_{0}(\delta-\gamma)}{A}$. Обозначим числитель этой формулы через $D$, т. е.

$$
\begin{gathered}
D=\alpha y_{0}-\frac{1}{2} x_{0}(\delta-\gamma), \\
C=\frac{D}{B} \cdot \frac{B}{\left(p-\frac{1}{2}(\delta+\gamma)\right)^{2}-B^{2}}==\frac{D}{B} e^{\frac{1}{2}(\delta+\gamma) t} \cdot \operatorname{sh} B t .
\end{gathered}
$$

Таким образом, с учетом (6) и (7) в случае 1$)$ для $X(p)$ получаем оригинал:

$$
x(t)=x_{0} e^{\delta t}+\alpha y_{0} t e^{\delta t}=e^{\delta t}\left(x_{0}+\alpha y_{0} t\right) ;
$$

для случая 2) на основе (9) и (11) получаем:

$$
x(t)=x_{0} e^{\frac{1}{2}(\delta+\gamma) t} \operatorname{ch} B t+\frac{D}{B} e^{\frac{1}{2}(\delta+\gamma) t} \operatorname{sh} B t=e^{\frac{1}{2}(\delta+\gamma) t}\left(x_{0} \operatorname{ch} B t+\frac{D}{B} \operatorname{sh} B t\right),
$$

здесь $B$ и $D$ определяются, соответственно, по формулам (5) и (10).

Аналогично для $Y(p)$ получаем оригинал:

для случая 1)

для случая 2)

$$
y(t)=e^{\gamma t}\left(y_{0}+\beta x_{0} t\right)
$$

$$
y(t)=e^{\frac{1}{2}(\delta-\gamma) t}\left(y_{0} \operatorname{ch} B t+\frac{D_{1}}{B} \operatorname{sh} B t\right) .
$$

Здесь $D_{1}=\beta x_{0}+\frac{1}{2} y_{0}(\delta-\gamma), B$ определяется по формуле (5).

Таким образом, получено решение системы дифференциальных уравнений (1) операторным методом.

\section{Результаты и обсуждение}

Для пояснения разработанного метода рассмотрим условный пример. Пусть $t_{0}=1$, $x_{0}=50000, y_{0}=45000, \alpha=0,05, \beta=0,03, \gamma=-0,02, \delta=-0,01$.

На рис. 1 построены графики изменения численностей $x$ и $y$ со временем. 


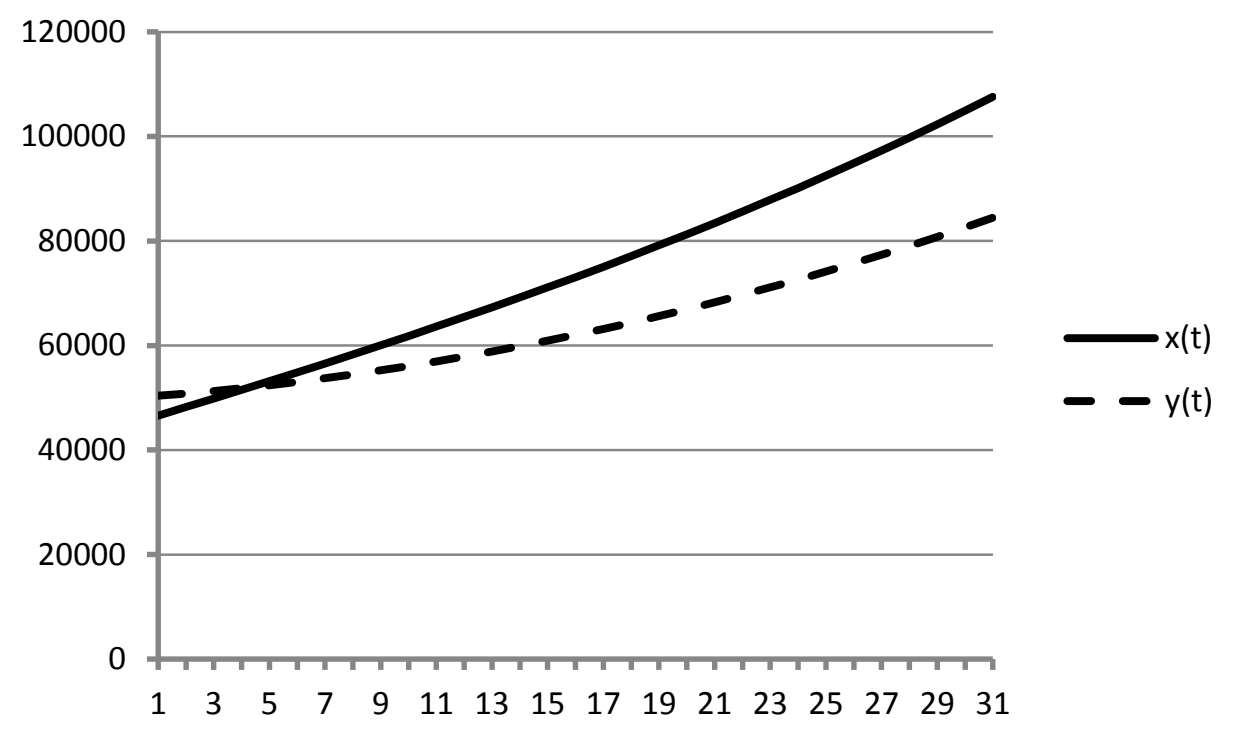

Рис. 1. Графики изменения численностей $x$ и $y$ со временем

Fig. 1. Graphs of changes in numbers $\mathrm{x}$ and $\mathrm{y}$ over time

Проанализируем частный случай разработанной математической модели. Если $x^{\prime}\left(t_{0}\right)=0$ и $y^{\prime}\left(t_{0}\right)=0$, то из (1) следует, что

$$
\left\{\begin{array}{l}
\alpha=-\gamma \frac{x_{0}}{y_{0}}, \\
\beta=-\delta \frac{y_{0}}{x_{0}}, \\
\alpha \cdot \beta=\gamma \cdot \delta .
\end{array}\right.
$$

В этом случае

$$
\begin{aligned}
& x(t)=e^{\frac{1}{2}(\delta+\gamma) t} x_{0}\left(\operatorname{ch} \frac{1}{2}(\delta+\gamma) t-\operatorname{sh} \frac{1}{2}(\delta+\gamma) t\right) \\
& y(t)=e^{\frac{1}{2}(\delta+\gamma) t} y_{0}\left(\operatorname{ch} \frac{1}{2}(\delta+\gamma) t-\operatorname{sh} \frac{1}{2}(\delta+\gamma) t\right) .
\end{aligned}
$$

Нетрудно показать, что производные первого порядка этих функций равны нулю, т. е. графики представляют собой параллельные прямые линии.

Наиболее близкими к разработанной модели являются модели, рассмотренные в статьях [Канищева, 2017, Руренко, 2017, Фирсова, Глухова, 2015]. В статье [Канищева, 2017] для получения решения анализируются условия равновесия системы дифференциальных уравнений, сама система не решается. В работе [Руренко, 2017] модель Ричардсона приводится к нелинейной управляемой системе дифференциальных уравнений. Для нахождения структуры оптимального управления строится гамильтониан и применятся принцип максимума Понтрягина. При применении такого математического аппарата модель становится очень сложной. Полученные решения, также как и в настоящем исследовании, содержат гиперболические функции. Громоздкость математического аппарата и отсутствие конкретного числового примера затрудняет анализ результатов данной работы. Решение модели Ричардсона, полученное в статье [Фирсова, Глухова, 2015] также основывается на устойчивости системы дифференциальных уравнений. В статье [Фирсова, Глухова, 2015]предложен метод задания коэффициентов системы. 
Рассмотрение наиболее близких к разработанной в данном исследовании модели позволяет сделать вывод, что используемый в статье методологический подход является новым, наглядным и эффективным.

Дальнейшим направлением исследований является разработка модели многопартийной конкуренции (число партий больше двух), использование нечетких чисел в качестве коэффициентов системы. Представляет интерес рассмотрение коалиционной политики партий [Сидоров, 2016].

\section{Заключение}

Таким образом, в настоящей работе получены следующие результаты:

1) для описания конкуренции политических партий использована модифицированная модель Ричардсона в виде системы двух дифференциальных уравнений с четырьмя коэффициентами;

2) получено аналитическое решение динамической системы дифференциальных уравнений с помощью операторного метода;

3) для проверки разработанного алгоритма рассмотрен конкретный числовой пример;

4) проанализированы особенности применения и перспективы дальнейшего развития нового разработанного метода описания конкуренции политических партий.

Следует отметить, что выводы, полученные от математических моделей социальноэкономических процессов, следует проверить эмпирически в реальных условиях.

\section{Список литературы}

1. Андрианова Е.Г., Головин С.А., Зыков С.В., Лесько С.А., Чукалина Е.Р. 2020. Обзор современных моделей и методов анализа временных рядов динамики процессов в социальных, экономических и социотехнических системах. Российский технологический журнал, 8 (4): 7-45. DOI https://doi.org/10.32362/2500-316X-2020-8-4-7-45.

2. Гильманов В.В. 2017. Партийные модели рекрутирования правящих элит и лидеров. Научные ведомости Белгородского государственного университета. Серия: История. Политология, 22 (271): 189-199.

3. Канищева О.И. 2017. Применение математических средств при моделировании военных конфликтов. Актуальные направления научных исследований XXI века: теория и практика, 8-1 (34-1): 200-204.

4. Михайлов А.П., Петров А.П., Прончева О.Г., Маревцева Н.А. 2017. Модель информационного противоборства в социуме при периодическом дестабилизирующем воздействии. Математическое моделирование, 29 (2): 23-32.

5. Остапенко В.В., Остапенко О.С., Беляева Е.Н., Ступницкая Ю.В. 2012. Математические модели борьбы партий за электорат или компаний за рынки сбыта. Кибернетика и системный анализ, 48 (6): 11-19.

6. Петров А.П., Прончева О.Г. 2019. Моделирование выбора позиций индивидами при информационном противоборстве с двухкомпонентной повесткой. Математическое моделирование, 31 (7): 91-108.

7. Петров А.П., Маслов А.И., Цаплин Н.А. 2015. Моделирование выбора позиций индивидами при информационном противоборстве в социуме. Математическое моделирование, 27 (12): 137-148.

8. Руренко Е.Н. 2017. Модель Ричардсона с управлением в одном частном случае. Advanced Science, 3: 123-129.

9. Сидоров В.В. 2016. Коалиционная политика политических партий в парламентских системах. Казань: Казан. ун-т, 149.

10. Фирсова И., Глухова Е. 2015. Математическая модель отношений между двумя государствами для решения проблем вооруженного конфликта. Финансовая жизнь, 1: 25-28.

11. Comissiong D.M.G., Sooknanan J. 2018. A review of the use of optimal control in social models. Internat. J. of Dynamics and Control, 6 (4): 1841-1846. 
12. Dominioni G., Marasco A., Romano A. 2018. A mathematical approach to study and forecast racial groups interactions: deterministic modeling and scenario method, 52: 1929-1956. DOI doi.org/10.1007/ s11135-017-0581-9.

13. Kubiv S. Balanyuk Y.Copyright, Kubiv S., Balanyuk Y. 2020. Development of a mathematical model of conflict between the parties in the implementation of the offset transaction. Technology audit and production reserves, Socionet; Technology audit and production reserves, 2(4(52)): 28-31. DOI 10.15587/ 2312-8372.2020.201260.

14. Marevtseva N.A. 2017. Model' informatsionnogo protivoborstva v sotsiume pri periodicheskom destabiliziruyushchem vozdeistvii. Matematicheskoe modelirovanie, 29 (2): 23-32.

15. Misra A.K., Kumar A. 2012. A simple mathematical model for the spread of two political parties. Nonlinear Analysis: Modelling and Control, 17(3): 343-354.

16. Nyabadza F., Alassey T.Y., Muchatibaya G. 2016. Modelling the dynamics of two political parties in the presence of switching. SpringerPlus, 5: 1018. DOI https://doi.org/10.1186/s40064-016-2483-z.

17. Petukhov A.Yu., Malkhanov A.O., Sandalov V.M., Petukhov Yu.V. 2018. Modeling conflict in a social system using diffusion equations Simulation, 94 (12): 1053-1061. DOI https://doi.org/10.1177/ 0037549718761573.

18. Rinaldi S., Della Rossa F. 2018. Conflicts among N armed groups: scenarios from a new descriptive model. Nonlinear Dynamics, 92 (3): 3-12. DOI doi.org/10.1007/s11071-017-3446-9.

19. Tsybulin V.G., Khosaeva Z.K. 2019. Mathematical model of political differentiation under social tension. Computer Research and Modeling, 11 (5): 999-1012. DOI10.20537/2076-7633-2019-11-5-999-1012.

20. Winkel B. 2017. 2012-Misra-Mathematics Model for the Spread of Two Political Parties. https://www.simiode.org/resources/4091.

\section{References}

1. Andrianova E.G., Golovin S.A., Zykov S.V., Les'ko S.A., Chukalina E.R. 2020. Obzor sovremennykh modeley i metodov analiza vremennykh ryadov dinamiki protsessov $\mathrm{v}$ sotsial'nykh, ekonomicheskikh i sotsiotekhnicheskikh sistemakh [Review of modern models and methods for analyzing time series of process dynamics in social, economic, and sociotechnical systems]. Rossiyskiy tekhnologicheskiy zhurnal [[Russian Technological Journal]], 8 (4): 7-45. DOI https://doi.org/10.32362/ 2500-316X-2020-8-4-7-45.

2. Gil'manov V.V. 2017. Partiynyye modeli rekrutirovaniya pravyashchikh elit i liderov [Party models for recruiting ruling elites and leaders]. Nauchnyye vedomosti Belgorodskogo gosudarstvennogo universiteta. Seriya: Istoriya. Politologiya [Belgorod State University Scientific bulletin. Issues Via in tempore. History and political science], 22 (271): 189-199.

3. Kanishcheva O.I. 2017. Primeneniye matematicheskikh sredstv pri modelirovanii voyennykh konfliktov [The use of mathematical tools in the modeling of military conflicts]. Aktual'nyye napravleniya nauchnykh issledovaniy XXI veka: teoriya i praktika [Current Directions of Scientific Research of the XXI Century: Theory and Practice], 8-1 (34-1): 200-204.

4. Mikhaylov A.P., Petrov A.P., Proncheva O.G., Marevtseva N.A. 2017. Model' informatsionnogo protivoborstva $\mathrm{v}$ sotsiume pri periodicheskom destabiliziruyushchem vozdeystvii [The model of information warfare in society with periodic destabilizing effects]. Matematicheskoye modelirovaniye [Mathematical modeling], 29 (2): 23-32.

5. Ostapenko V.V., Ostapenko O.S., Belyayeva E.N., Stupnitskaya YU.V. 2012. Matematicheskiye modeli bor'by partiy za elektorat ili kompaniy za rynki sbyta [Mathematical models of the struggle of parties for the electorate or companies for sales markets]. Kibernetika i sistemnyy analiz [Cybernetics and System Analysis], 48 (6): 11-19.

6. Petrov A.P., Proncheva O.G. 2019. Modelirovaniye vybora pozitsiy individami pri informatsionnom protivoborstve $\mathrm{s}$ dvukhkomponentnoy povestkoy [Modeling the choice of positions by individuals in an information confrontation with a two-component agenda]. Matematicheskoye modelirovaniye [Mathematical modeling], 31 (7): 91-108.

7. Petrov A.P., Maslov A.I., Tsaplin N.A. 2015. Modelirovaniye vybora pozitsiy individami pri informatsionnom protivoborstve $\mathrm{v}$ sotsiume [Modeling of the choice of positions by individuals in the information confrontation in society]. Matematicheskoye modelirovaniye [Mathematical modeling], 27 (12): 137-148. 
8. Rurenko E.N. 2017. Model' Richardsona s upravleniyem v odnom chastnom sluchaye [The Richardson model with control in one particular case]. Advanced Science, 3: 123-129.

9. Sidorov V.V. 2016. Koalitsionnaya politika politicheskikh partiy v parlamentskikh sistemakh [Coalition politics of political parties in parliamentary systems]. Kazan': Kazan. un-t, 149.

10. Firsova I., Glukhova E. 2015. Matematicheskaya model' otnosheniy mezhdu dvumya gosudarstvami dlya resheniya problem vooruzhennogo konflikta [A mathematical model of relations between two States for solving problems of armed conflict]. Finansovaya zhizn' [Financial Life], 1: 25-28.

11. Comissiong D.M.G., Sooknanan J. 2018. A review of the use of optimal control in social models. Internat. J. of Dynamics and Control, 6 (4): 1841-1846.

12. Dominioni G., Marasco A., Romano A. 2018. A mathematical approach to study and forecast racial groups interactions: deterministic modeling and scenario method, 52: 1929-1956. DOI doi.org/10.1007/ s11135-017-0581-9.

13. Kubiv S. Balanyuk Y.Copyright, Kubiv S., Balanyuk Y. 2020. Development of a mathematical model of conflict between the parties in the implementation of the offset transaction. Technology audit and production reserves, Socionet; Technology audit and production reserves, 2(4(52)): 28-31. DOI 10.15587/ 2312-8372.2020.201260.

14. Marevtseva N.A. 2017. Model' informatsionnogo protivoborstva v sotsiume pri periodicheskom destabiliziruyushchem vozdeistvii. Matematicheskoe modelirovanie, 29 (2): 23-32.

15. Misra A.K., Kumar A. 2012. A simple mathematical model for the spread of two political parties. Nonlinear Analysis: Modelling and Control, 17(3): 343-354.

16. Nyabadza F., Alassey T.Y., Muchatibaya G. 2016. Modelling the dynamics of two political parties in the presence of switching. SpringerPlus, 5: 1018. DOI https://doi.org/10.1186/s40064-016-2483-z.

17. Petukhov A.Yu., Malkhanov A.O., Sandalov V.M., Petukhov Yu.V. 2018. Modeling conflict in a social system using diffusion equations Simulation, 94 (12): 1053-1061. DOI https://doi.org/10.1177/ 0037549718761573.

18. Rinaldi S., Della Rossa F. 2018. Conflicts among N armed groups: scenarios from a new descriptive model. Nonlinear Dynamics, 92 (3): 3-12. DOI doi.org/10.1007/s11071-017-3446-9.

19. Tsybulin V.G., Khosaeva Z.K. 2019. Mathematical model of political differentiation under social tension. Computer Research and Modeling, 11 (5): 999-1012. DOI10.20537/2076-7633-2019-11-5-999-1012.

20. Winkel B. 2017. 2012-Misra-Mathematics Model for the Spread of Two Political Parties. https://www.simiode.org/resources/4091.

\section{ИНФОРМАЦИЯ ОБ АВТОРАХ}

Ганичева Антонина Валериановна, кандидат физико-математических наук, доцент, доцент кафедры физико-математических дисциплин и информационных технологий Тверской государственной сельскохозяйственной академии, г. Тверь, Россия

Ганичев Алексей Валерианович, доцент кафедры информатики и прикладной математики Тверского технического университета, г. Тверь, Россия

\section{INFORMATION ABOUT THE AUTHORS}

Antonina V. Ganicheva, Candidate of Physical And Mathematical Sciences, Associate Professor, Department Physical And Mathematical Disciplines And Information Technology, Federal State Budgetary Educational Institution Of Higher Education «Tver State Agricultural Academy», Tver, Russian Federation

Aleksey V. Ganichev, Associate Professor, Department Of Informatics And Applied Mathematics, Federal State Budgetary Educational Institution Of Higher Education «Tver State Technical University», Tver, Russian Federation 